\title{
New aspects of microbial vitamin K2 production by expanding the product spectrum
}

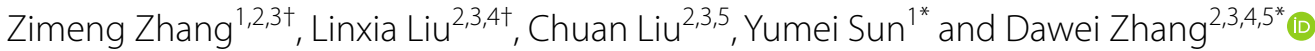

\begin{abstract}
Vitamin K2 (menaquinone, MK) is an essential lipid-soluble vitamin with critical roles in blood coagulation and bone metabolism. Chemically, the term vitamin K2 encompasses a group of small molecules that contain a common naphthoquinone head group and a polyisoprenyl side chain of variable length. Among them, menaquinone-7 (MK-7) is the most potent form. Here, the biosynthetic pathways of vitamin K2 and different types of MK produced by microorganisms are briefly introduced. Further, we provide a new aspect of MK-7 production, which shares a common naphthoquinone ring and polyisoprene biosynthesis pathway, by analyzing strategies for expanding the product spectrum. We review the findings of metabolic engineering strategies targeting the shikimate pathway, polyisoprene pathway, and menaquinone pathway, as well as membrane engineering, which provide comprehensive insights for enhancing the yield of MK-7. Finally, the current limitations and perspectives of microbial menaquinone production are also discussed. This article provides in-depth information on metabolic engineering strategies for vitamin K2 production by expanding the product spectrum.
\end{abstract}

Keywords: Vitamin K2, Biosynthetic pathway, Pathway engineering, Product spectrum

\section{Introduction}

Dr. Henrik Dam discovered vitamin $K$ in the 1930s and shared the 1943 Nobel Prize in medicine with Edward Doisy for their work on this fat-soluble bioactive compound [1-3]. Vitamin $\mathrm{K}$ serves as a cofactor for $\gamma$-glutamyl carboxylase (GGCX), which converts glutamic acid residues of vitamin K-dependent proteins (VKDPs) into $\gamma$-carboxyglutamic acid (Gla). There are more than 15 types of VKDPs, including coagulation factors II (prothrombin), VII, IX, and X, as well as the anticoagulation factors $\mathrm{C}$, protein $\mathrm{S}$, and osteocalcin [4-7]. Additionally, optimal vitamin $\mathrm{K}$ status is strongly associated with various health benefits, such as preventing or

\footnotetext{
*Correspondence: sunmei62@163.com; zhang_dw@tib.cas.cn

${ }^{\dagger}$ Zimeng Zhang and Linxia Liu contributed equally to this work

${ }^{1}$ School of Biological Engineering, Dalian Polytechnic University, Dalian 116034, China

${ }^{2}$ Key Laboratory of Systems Microbial Biotechnology, Chinese Academy of Sciences, Tianjin 300308, China

Full list of author information is available at the end of the article
}

alleviating cardiovascular disease, osteoporosis, osteoarthritis, cancer, inflammatory diseases, diabetes, chronic kidney disease, immune disorders, and Alzheimer's disease $[4,7-10]$. Perhaps unsurprisingly, vitamin $\mathrm{K}$ deficiency may also influence the mortality and morbidity of COVID-19 patients [11].

The term vitamin $\mathrm{K}$ denotes a series of fat-soluble compounds that contain a 2-methyl-1,4-naphthoqumone moiety as the basic skeleton and an isoprenoid chain at the 3-position [12]. Based on the side-chain structure, vitamin $\mathrm{K}$ isoforms are categorized as vitamin $\mathrm{K} 1$ (phylloquinone, PK), vitamin K2 (menaquinone-n, MK-n) and vitamin K3 (menadione, MD) (Fig. 1) [13-15]. Vitamin $\mathrm{K} 3$ is a synthetic product without a side chain, while vitamin $\mathrm{K} 1$ and $\mathrm{K} 2$ occur naturally. Vitamin $\mathrm{K} 1$ has an aliphatic side chain and is predominately found in various leafy green vegetables, fruits, and plant oils $[13,16]$. Vitamin K2 contains an unsaturated aliphatic side chain with a variable number (n) of 4 to 13 isoprene units and is referred to as MK-n [17]. Vitamin K1 is currently produced via chemical synthesis, and there is little evidence

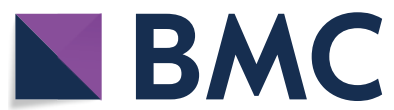

(c) The Author(s) 2021. This article is licensed under a Creative Commons Attribution 4.0 International License, which permits use, sharing, adaptation, distribution and reproduction in any medium or format, as long as you give appropriate credit to the original author(s) and the source, provide a link to the Creative Commons licence, and indicate if changes were made. The images or other third party material in this article are included in the article's Creative Commons licence, unless indicated otherwise in a credit line to the material. If material is not included in the article's Creative Commons licence and your intended use is not permitted by statutory regulation or exceeds the permitted use, you will need to obtain permission directly from the copyright holder. To view a copy of this licence, visit http://creativeco mmons.org/licenses/by/4.0/. The Creative Commons Public Domain Dedication waiver (http://creativecommons.org/publicdomain/ zero/1.0/) applies to the data made available in this article, unless otherwise stated in a credit line to the data. 

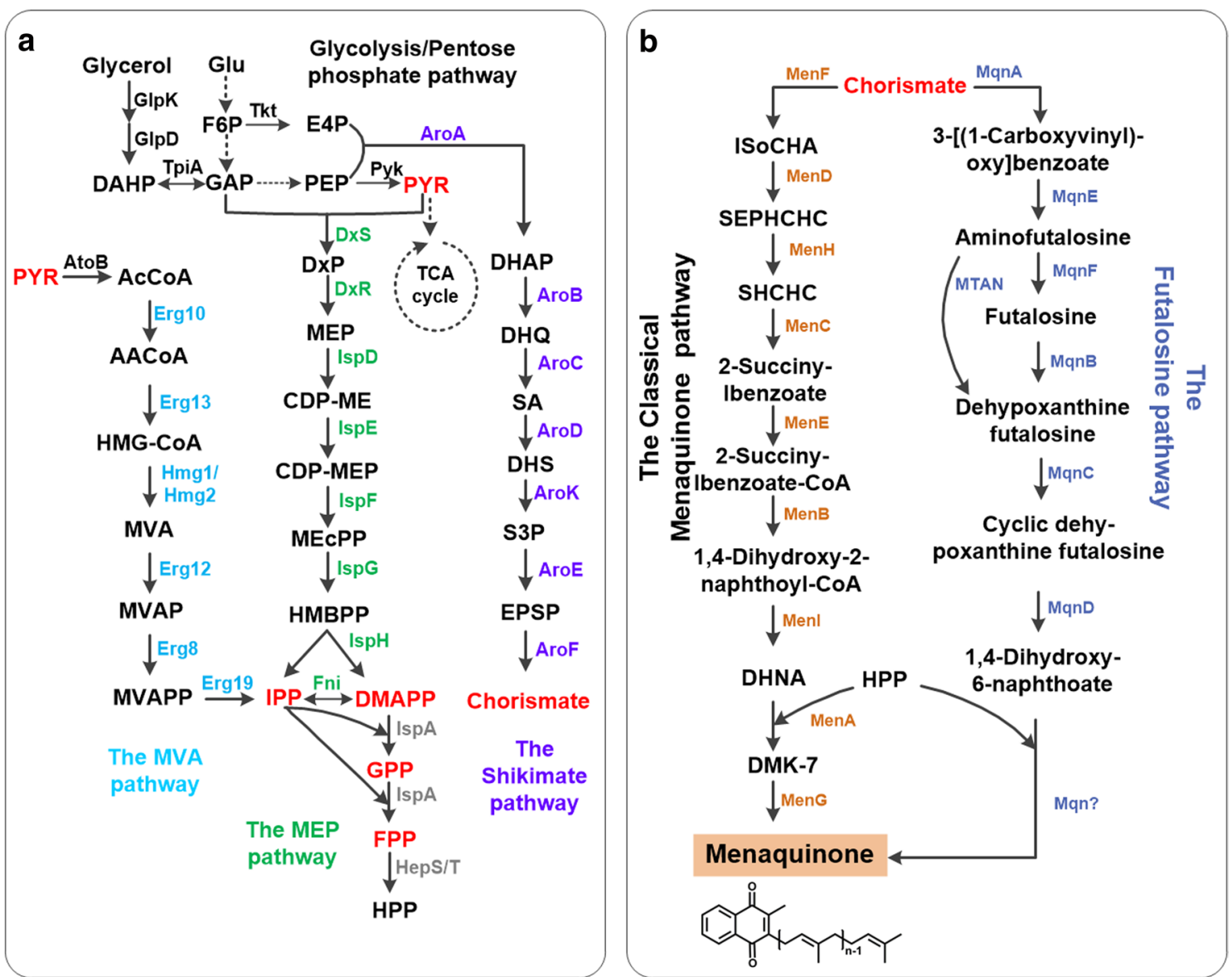

Fig. 1 The biosynthesis pathways of menaquinone in isoforms from Bacillus subtilis, Saccharomyces cerevisiae and S. coelicolor [26, 37, 41]. Menaquinone biosynthesis is a complex process involving multiple metabolic pathways, such as glycolysis, the pentose phosphate pathway, the shikimate pathway, the MEP or MVA pathway, as well as the classical MK pathway or futalosine pathway. a The important intermediates chorismate and polyisoprene are synthesized from the precursors G3P, pyruvate and E4P. $\mathbf{b}$ The classical pathway and the futalosine pathway for the biosynthesis of menaquinone. Enzymes are displayed in different colors in different pathways. Red typeface indicates several important intermediate metabolites

of successful attempts to reduce or eliminate the utilization of toxic chemicals in this process by applying enzymes $[13,16]$. However, consumers are increasingly favoring "natural" products like vitamin K2 fermented by Generally Recognized as Safe (GRAS) bacteria [18, 19]. Thus, this review focuses on strategies for industrial vitamin $\mathrm{K} 2$ biosynthesis.

Among vitamin $\mathrm{K} 2$ homologues, MK-4 is the most common form in animals, with the widest range of physiological activities [6]. The long-chain MK isoforms, such as MK-7, are found in fermented foods or produced by bacteria. The traditional Japanese food natto, which is made from soybeans fermented by Bacillus subtilis natto, contains MK-7 at a very high concentration [1]. MK-4 and MK-7 are allowed in the United States as nutritional supplements for bone health [20]. However, the administration of MK-4 is not reflected in an increased serum concentration [6]. By contrast, MK-7 is absorbed efficiently, which is reflected in increased serum MK-7 levels up to several days, thereby contributing to vitamin K status [6, 21]. US Pharmacopeia monographs have been developed to establish quality standards for menaquinone-7 as a dietary ingredient at typically recommended levels [22]. Although, there are cis, trans, and cis/trans isomers of MK-7, only the all-trans form is biologically active [21]. It can be produced naturally through fermentation, as in the case of natto, or by organic synthesis. In the process of organic synthesis of MK-7, cis/trans isomers can be produced at the same time. Thus, the potential for the different isomeric forms of MK-7 to have different cytotoxic or toxic properties is an important consideration [21, 23]. Naturally derived MK-7 is 
produced via fermentation of generally recognized as safe (GRAS) bacterial strains such as B. subtilis natto, and is therefore considered to be nontoxic [2]. Thus, enhancing the production of vitamin $\mathrm{K} 2$ by environmentally friendly fermentation has been extensively studied.

The aim of this review is to offer new insights for improving microbial factories for the production of vitamin K2, with a particular focus on pathway engineering and membrane engineering by expanding the product spectrum. The bacterial MK biosynthesis pathway and the producing microorganism that synthesize different dominant MK isoforms (MK-4, MK-6, MK-7, MK-8, etc.) are briefly discussed. Finally, the limitations of microbial menaquinone production are also discussed.

\section{Biosynthetic pathway of vitamin K2}

The selection of high-yield strains is based on the biosynthesis pathway of vitamin K2 in prokaryotes. In 1982, Bentley et al. [24] initially clarified the mechanism of vitamin $\mathrm{K} 2$ biosynthesis in bacteria and reviewed the detailed roles of shikimate, 2-succinylbenzoate, 1,4-dihydroxy-2-naphthoate, and other intermediates. They also described individual reactions in the pathway from the perspective of chemical mechanisms and enzymological characteristics [24]. The chemical structure of vitamin $\mathrm{K} 2$ includes a naphthoquinone ring and an isoprene side chain [25].

The naphthoquinone ring can be synthesized via the classical MK pathway or the futalosine pathway (Fig. 1) [26-28]. The classical MK pathway has been known for decades and is found in almost all aerobic or facultatively anaerobic prokaryotes. By contrast, the futalosinedependent pathway was first discovered and validated in S. coelicolor A3(2) in 2008, and is found in a broader taxonomic range of organisms, including also anaerobic microorganisms [29-31]. Both the classical MK pathway and the futalosine pathway diverge at chorismate and re-converge after the formation of menaquinone [32]. Therefore, chorismate is the key intermediate for the biosynthesis of the menaquinone ring. Chorismate is synthesized via the shikimate pathway from two precursors: E4P, which is an intermediate product of the pentose phosphate pathway, and phosphoenolpyruvate (PEP), an intermediate product of the glycolysis pathway or glycerol metabolism (Fig. 1a) [33, 34]. In the first step of the classical MK pathway, MenF converts chorismate into isochorismate, which is further converted by six enzymes encoded by the menDHCEB operon to form 1,4-dihydroxy-2-naphthoate (Fig. 1b) [17, 26]. In the first step of the futalosine pathway, MqnA catalyzes the dehydration of chorismate to generate 3-[(1-carboxyvinyl) oxy]-benzoic acid, which is then converted into 1,4-dihydroxy-6-naphthoate via three enzymatic reactions catalyzed by MqnBCD (Fig. 1b) [31, 35, 36].

Secondly, the isoprene side chain is generally produced from two simple five-carbon units, isopentenyl diphosphate (IPP) and dimethylallyl diphosphate (DMAPP), either via the 2-C-methyl-D-erythritol4-phosphate (MEP) pathway or the mevalonate (MVA) pathway $[37,38]$. The MVA pathway is present in most eukaryotes, archaea, some bacteria, as well as cytosol and mitochondria of plants, while most bacteria, chloroplasts, and apicomplexan parasites synthesize IPP and DMAPP through the MEP pathway [37, 39]. The MEP pathway consists of eight enzyme-catalyzed reactions involved in the formation of IPP and DMAPP. Glyceraldehyde 3-phosphate (G3P) and pyruvate from the glycolysis pathway or glycerol metabolism are converted by 1-deoxy-D-xylulose-5-phosphate synthase (Dxs) to form 1-deoxy-D-xylulose-5-phosphate (DXP), which enters the MEP pathway. Then, DXP can be converted into MEP by 1-deoxy-D-xylulose 5-phosphate reductoisomerase (Dxr). IPP and DMAPP are produced by a pathway containing five enzymes, called IspDEFGH, but DMAPP can also be converted into IPP by isopentenyl-diphosphate delta-isomerase (Fni) (Fig. 1a) [40]. The MEP pathway starts with the condensation of G3P and pyruvate, while the MVA pathway uses acetyl-CoA as a substrate. Acetyl-CoA is converted into acetoacetyl-CoA by acetyl-CoA acetyltransferase [41]. Then, the five enzymes Erg13, Hmg1/Hmg2, Erg12, Erg8, and Erg19 convert acetoacetyl-CoA to IPP. Finally, IPP undergoes a twostep catalytic reaction to form the isoprene side chainpolyprenyl-PP (C40) [41]. These two pathways have been widely used in studies of isoprene biosynthesis.

Finally, the membrane-bound enzymes polyprenyltransferase (MenA) and methyltransferase (MenG) combine the menaquinone ring and isoprene side chain to synthesize vitamin K2 [40].

\section{Different isoforms of vitamin $\mathrm{K} 2$ produced by various bacteria}

In the Western diet, cheese and curd produced with Lactococcus lactis are the most important sources of vitamin K2. Bøe and Holo [42] co-overexpressed $m v k$, preA, and $m e n A$ to increase the vitamin K2 yield (mainly MK-3, MK-7, and MK-9) (Table 1). When milk was fermented using the modified K2-overproducing strains, the vitamin K2 yield was effectively increased threefold compared to the wild type, which provides a foundation for the development of strains to ferment food with increased functional value [42].

MK-4 is found in small amounts in animal products, such as eggs and meat, and it is also the major form $(>90 \%)$ of vitamin $\mathrm{K}$ found in animal tissues [9, 43]. 
Table 1 Metabolic engineering for the production of vitamin K2

\begin{tabular}{|c|c|c|c|c|c|c|}
\hline Vitamin K2 & Host & Pathway engineering & Strategies & Media & Titer & References \\
\hline MK-7 and MK-9 & L. lactis & $\begin{array}{l}\text { The MVA pathway, the } \\
\text { polyprenyl pathway and } \\
\text { the MK pathway }\end{array}$ & $\begin{array}{l}\text { Overexpression of } m v k, \\
\text { preA, and menA }\end{array}$ & M17 & $680 \mathrm{nmol} / \mathrm{L}$ & [42] \\
\hline MK-4 & B. subtilis & $\begin{array}{l}\text { The MVA and MEP } \\
\text { pathway, the polyprenyl } \\
\text { pathway and the MK } \\
\text { pathway }\end{array}$ & $\begin{array}{l}\text { Overexpression of menA, } \\
\text { menG, crtE, dxs, dxr, ispD- } \\
F, \text { mvaK1, mvaK2, mvaD, } \\
\text { mvaS, mvaA }\end{array}$ & Fermentation medium & $120.1 \pm 0.6 \mathrm{mg} / \mathrm{L}$ & [44] \\
\hline MK-4 & P.pastoris & The polyprenyl pathway & Expression of hsUBIAD1 & BMMY & $0.24 \mathrm{mg} / \mathrm{g} \mathrm{DCW}$ & {$[45]$} \\
\hline MK-7 & B. subtilis natto & - & $\begin{array}{l}\text { Optimization of growth } \\
\text { parameters }\end{array}$ & Fermentation medium & $12.09 \mathrm{mg} / \mathrm{L}$ & [55] \\
\hline MK-8 & E. coli & $\begin{array}{l}\text { The SA pathway, the } \\
\text { ubiquinone-8 pathway } \\
\text { and the MK pathway }\end{array}$ & $\begin{array}{l}\text { ubicA deletion, overexpres- } \\
\text { sion of menA and menD }\end{array}$ & LGN & $290 \mu \mathrm{g} / \mathrm{g}$ WCW & [46] \\
\hline MK-7 & E. coli & The SA pathway & $\begin{array}{l}\text { Overexpression aroA, aroK } \\
\text { and the feedback inhibi- } \\
\text { tion-resistant aroGfbr }\end{array}$ & & $19.1 \mathrm{mg} / \mathrm{L}$ & [64] \\
\hline MK-7 & B. subtilis & $\begin{array}{l}\text { The MEP pathway and the } \\
\text { MK pathway }\end{array}$ & $\begin{array}{l}\text { The expression of menA- } \\
d x s-d x r \text {-idi cassette }\end{array}$ & SYG & $50 \mathrm{mg} / \mathrm{L}$ & [75] \\
\hline MK-7 & E. coli & $\begin{array}{l}\text { The polyprenyl pathway } \\
\text { and the MVA pathway }\end{array}$ & $\begin{array}{l}\text { Overexpression of hepPPS } \\
\text { and lower expression of } \\
\text { mvaE, mvaS and mvK }\end{array}$ & $\begin{array}{l}\text { Minimal medium added } \\
\text { glucose }\end{array}$ & $2.3 \mu \mathrm{M}$ & [77] \\
\hline
\end{tabular}

$D C W$ dry cell weight, $W C W$ wet cell weight

MK-4 is mainly produced by microbial fermentation, which only produces the all-trans configuration, while the chemical synthesis of MK-4 remains a challeng [23]. Yuan et al. [44] improved the synthesis efficiency of MK-4 by combinatorial pathway engineering involving four modules, a MK-4 biosynthesis module (overexpression of menA, menG, and $c r t E$ ), MEP module (knockout of hepT; overexpression of $d x s, d x r$, and ispD-ispF), MVA isoprenoid module (heterogeneous expression of $m v a K 1, m v a K 2, m v a D, m v a S$, and $m v a A$ ), and menaquinone module (overexpression of menA and menG). The MK-4 of resulting engineered $B$. subtilis increased to $120.1 \mathrm{mg} / \mathrm{L}$ in shake flasks (Table 1) [44]. Another example is in the methylotrophic yeast Pichia pastoris, in which a novel synthetic pathway for the production of MK-4 was introduced via heterologous expression of UbiA prenyltransferase containing 1 (HsUBIAD1). After optimizing the expression conditions, the yield increased 4.37 times compared with that under the initial conditions (Table 1) [45].

Escherichia coli is a facultatively anaerobic bacterium that produces benzoquinone-type ubiquinone-8 (Q-8) under aerobic conditions and mainly synthesizes naphthoquinone-type menaquinone-8 (MK-8) under anaerobic conditions. Kong and Lee [46] enhanced the MK-8 content by modulating two precursors pools and blocking the competing synthesis of Q-8. Further, overexpression of menA and menD increased the MK- 8 yield fivefold compared with the wild-type (Table 1) [46].
Some enterobacteria such as Eubacterium lentum, Veillonella, Enterobacterium, and Bacteroides species can produce MK-6, MK7, MK-8, MK-10, and MK-11, but the exact mechanisms remain underexplored $[47,48]$.

The MK-7 long-chain isoform is an essential nutrient for humans because it is the most efficiently absorbed form of vitamin $\mathrm{K}$ and has a long half-life of $68 \mathrm{~h}$ compared with only $1-2 \mathrm{~h}$ for $\mathrm{K} 1[6,49,50]$. B. subtilis natto is the main host for the industrial production of vitamin $\mathrm{K} 2$ [51]. It was also shown to produce a range of vitamin $\mathrm{K} 2$ isoforms, from MK-4 to MK-8, but MK-7 accounts for more than $90 \%$ of the total [52]. B. subtilis natto was originally used in the fermentation of natto, a traditional Japanese food, which is an excellent source of MK-7 [53, 54]. Optimization of $B$. subtilis natto growth parameters including temperature, $\mathrm{pH}$, and agitation in a glycerolbased medium increased the MK-7 concentration to $12.09 \mathrm{mg} / \mathrm{L}$ in a biofilm reactor, without genetic modification [55]. Next, the review will focus on the metabolic engineering of microbes for the production of MK-7.

\section{Metabolic engineering of MK-7 production} from the aspects of pathways and the product spectrum In the pathways described above, the most important intermediates for vitamin $\mathrm{K} 2$ biosynthesis are chorismate and isoprene. Chorismate enters the classical MK pathway or the futalosine pathway to form the naphthoquinone head-group (Fig. 1) [40]. The polyisoprene tail is produced from two, five-carbon (C5) universal 
precursors: IPP and DMAPP through the MEP pathway or the MVA pathway [41]. Then, the polyisoprene tail is ligated with the naphthoquinone head by MenA to form demethylmenaquinone (DMK) and MK-7 is synthesized via the methylation of DMK [40]. Therefore, the key points of the pathways which may be the rate-limiting steps of vitamin K2 biosynthesis need to be discussed in depth. Undoubtedly, precursors for vitamin K2 biosynthesis also participate in the biosynthesis of various other chemicals, including some well-studied ones, such as terpenoids and aromatic acids. Therefore, this review not only discusses the unique metabolic engineering strategies for the production of vitamin $\mathrm{K} 2$ but also offers a perspective of engineering methods for other products that share the same precursors or pathways, thereby providing ideas for future research on vitamin K2.

\section{Metabolic engineering of the shikimate pathway to improve chorismate production}

The shikimate pathway connects the central carbon metabolism with the biosynthesis of chorismate, which is a key precursor for the production of aromatic amino acids and a large number of other aromatic compounds in plants and microorganisms, including vitamin K2 (Fig. 2) [56-58]. In E. coli, three different 3-deoxy-D-arabino-heptulosonate-7-phosphate synthases (DAHPS) isoenzymes encoded by the AroGFH genes contribute to the total DAHPS activity and are subject to allosteric

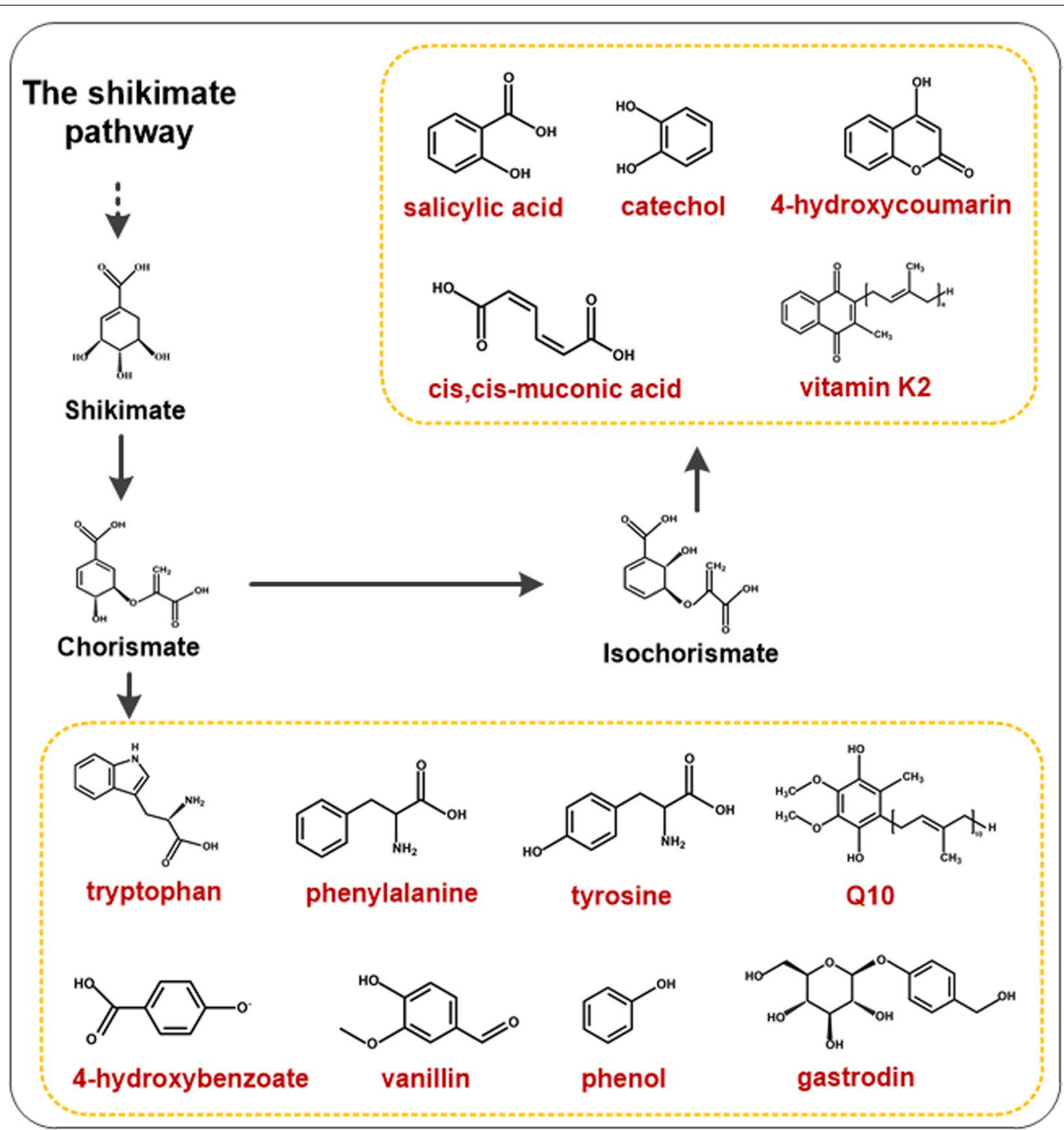

Fig. 2 The shikimate pathway and heterologous products derived from chorismate and isochorismate. Red typeface indicates the product spectrum derived from chorismate or isochorismate 
control by L-phenylalanine, L-tyrosine, and L-tryptophan, respectively [59-62]. Through structural analysis of mutant enzymes that are not sensitive to feedback, certain specific amino acid residues involved in the allosteric site have been identified, and feedback resistant (fbr) variants of AroG and AroF have been developed [59, 62]. Hence, elimination of feedback inhibition of the key enzymes obtained by introducing site-directed mutations is usually the first and most important step for the construction of a high-producing strain.

In a study of modular pathway engineering to promote MK-7 production, Yang et al. [63] found that overexpression of AroADE in B. subtilis inhibited the biosynthesis of MK-7, but the transcriptional levels of these genes were significantly increased ( $>300$-fold). It was suggested that increased production of aromatic amino acids resulted in feedback inhibition of the shikimate pathway, and ultimately inhibited the production of MK-7 [63]. Cui et al. [64] overexpressed aroAK together with the feedback inhibition-resistant aro $G^{\mathrm{fbr}}$ from $E$. coli, which resulted in a twofold increase of MK-7 production compared to the wild type (Table 1).

We can also learn from studies on the production of aromatic chemicals and derivatives such as $p$-aminobenzoate, salicylate, cis, cis-muconic acid (MA), 4-hydroxycoumarin (4-HC), 4-hydroxybenzoic acid, et cetera [65-68]. To inprove the microbial biosynthesis of 4-hydroxycoumarin, Lin et al. [69] constructed the chorismate-boosting plasmid pCS-APTA (overexpressing aroL, ppsA, tktA and aro $G^{\mathrm{fbr}}$ ), which led to the production of $283.9 \mathrm{mg} / \mathrm{L} 4 \mathrm{HC}$, a $37 \%$ increase compared with its original strain. Additionally, strategies for the synthesis of chorismate derivatives are based on the release of pyruvate competition in many cases. So, utilization of metabolic engineering strategies involving the pyruvate recycling system combined with improved chorismate supply can promote cell growth, leading to high productivity and yield of chorismate derivatives. Cui et al. [64] designed a bifunctional Phr60-Rap60-Spo0A quorumsensing molecular switch to fine-tune the expression of $p y k$, which limited the metabolic flux of pyruvate from PEP to the TCA cycle and enhanced its supply for MK-7 biosynthesis.

\section{Metabolic engineering of polyisoprene biosynthesis}

More than 50,000 isoprenoid compounds are found in nature [70, 71]. Isoprenoids are a diverse group of molecules found in all organisms, where they perform a wide variety of important biological functions including electron transport, hormonal signaling, antioxidation, and growth regulation [70, 72-74]. The isoprenoid side chain anchors quinones in the lipid membrane, while the quinone head is responsible for the electron transfer capacity [41]. The long isoprenoid side chains, such as geranyl pyrophosphate (GPP), farnesyl pyrophosphate (FPP), geranylgeranyl pyrophosphate (GGPP), or heptaprenyl diphosphate (HPP) are synthesized by the consecutive condensation of the five-carbon monomer IPP and its isomer DMAPP (Fig. 3) [26].

Most bacteria biosynthesize the precursors of isoprenoids via the MEP pathway and the yield of isoprenoids can be increased by introducing a heterologous MVA pathway, which can function in parallel with the native MEP pathway to provide IPP and DMAPP [37]. Yuan et al. [44] knocked out hepT while simultaneously overexpressing $d x s, d x r$, and $i s p D-i s p F$ in the MEP module, combine with the overexpression of the heterogeneous MVA module genes (mvaK1, mvaK2, mvaD, mvaS, and $m v a A$ ). This engineering strategy increased the MK-4 yield 11.1-fold to $90.1 \pm 1.7 \mathrm{mg} / \mathrm{L}$ compared with the parental strain (Table 1) [44].

Pathway engineering of polyisoprene biosynthesis is also commonly used to improve the productivity of vitamin K2. The MEP pathway for the biosynthesis of IPP and DMAPP consists of eight enzyme-catalyzed reactions. Ma et al. [75] increased the titer of rate-limiting enzymes in the MEP pathway by the expressing the Pspac-MenA-DxS-Dxr-Idi cassette, which resulted in an 11-fold increase of MK-7 production (Table 1). Yang et al. [63] improved the supply of heptaprenyl-PP by engineering the MEP pathway via overexpression of $d x s, d x r$, ispD $($ yacM), and $i s p F(y a c N)$. However, overexpression of the other three pathway genes ispE, ispH (yqfP), and ispA (yqiD) resulted in a decrease in the production of MK-7 without a clear explanations [63]. However, another study also optimized the MEP pathway to enhance MK-7 production. In this study, the ispDFHG genes were sequentially overexpressed in the BS20 strain, which intrinsically overexpresses $d x s$ and $d x r$. The highest MK-7 titer of the resulting strain reached $415 \pm 3.2 \mathrm{mg} / \mathrm{L}$, representing a $29 \%$ increase over the original strain [76]. It is suggested that $i s p E$ overexpression might lead to an imbalance in the MEP pathway and the expression order of the genes had an impact on the MK-7 yield, which should be kept in mind when attempting to construct high-yield strains.

The MVA pathway does not exist in most prokaryotes, but a heterologous MVA pathway can be constructed in bacterial hosts. Gao et al. [77] developed an engineered $E$. coli strain for MK-7 production by introducing HepPPS (heptaprenyl pyrophosphate synthetase) from B. subtilis and optimizing the enzyme expression of the MVA pathway. This strategy increased the titer of MK-7 to $2.3 \mu \mathrm{M}$, which was 22 -fold higher than that of the original strain (Table 1) [77].

Furthermore, the length of the isoprenoid side chain and the type of quinone differ among species [78]. For 


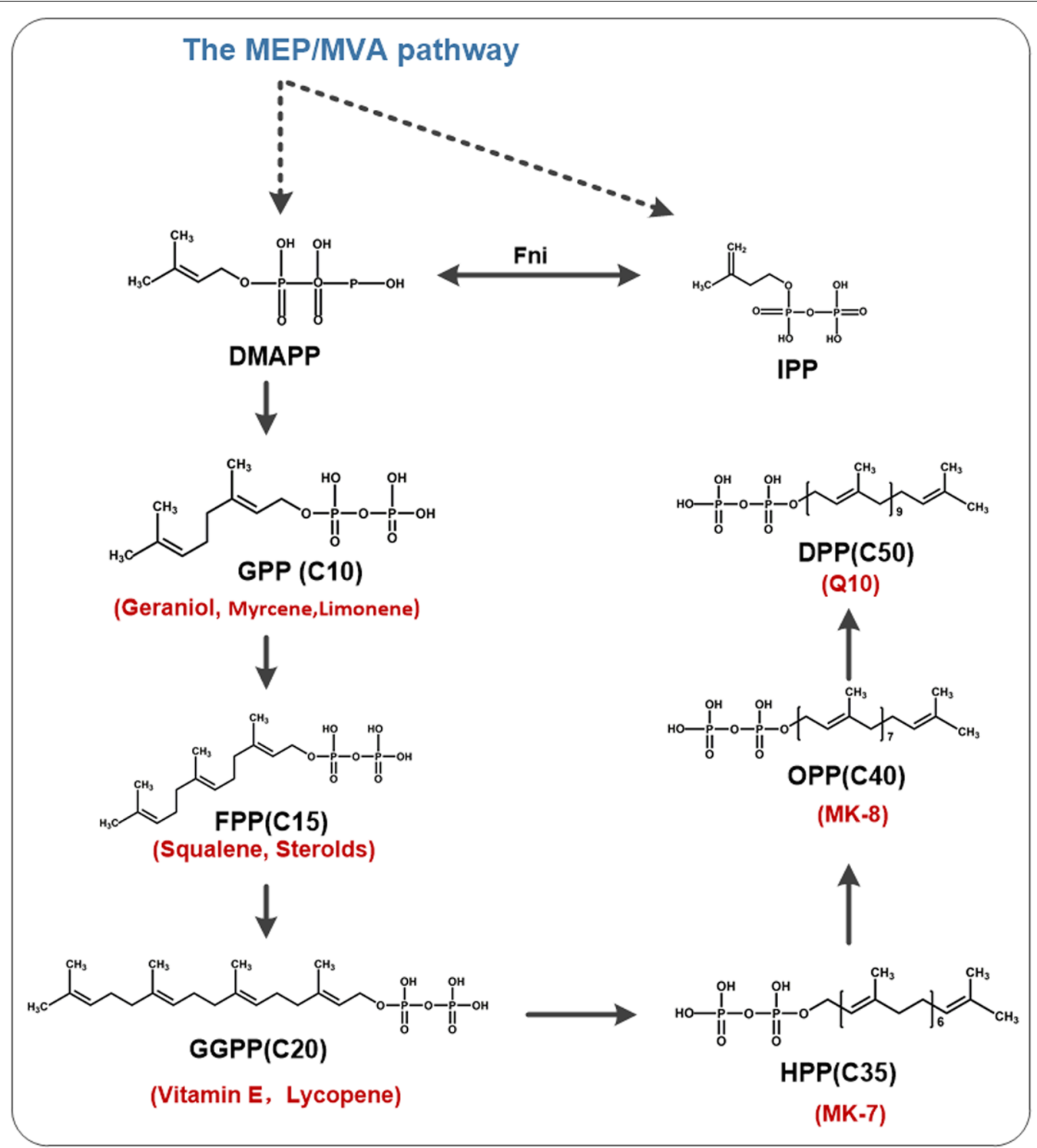

Fig. 3 Isoprenoid-derived molecules and the biosynthesis of isoprenoids, including the formation of C 5 building blocks IPP and DMAPP by the MEP pathway and the MVA pathway. Red typeface indicates the spectrum of products derived from polyisoprene

example, bacteria such as B. subtilis produce MK7, E. coli synthesize UQ8 and MK8, while yeasts such as Saccharomyces cerevisiae and Schizosaccharomyces pombe produce UQ6 and UQ10, respectively [41]. Notably, the isoprenoid pathway can be expanded to produce more diverse compounds such as limonene, lycopene, or vitamin E, and related studies offer great inspiration for the engineering of microbial factories for vitamin $\mathrm{K} 2$ production (Fig. 3) [38, 41, 79-83].

Additionally, increasing the precursor pools of pyruvate and G3P or acetyl-CoA is an important strategy for enhancing the synthesis of polyisoprenes. Yang et al. overexpressed $g l p D$ from the glycerol metabolism pathway in B. subtilis 168, which increased the yield of MK-7 to $13.7 \pm 0.2 \mathrm{mg} / \mathrm{L}$ [63]. In the case of lycopene, which is also a terpenoid like vitamin K2, Farmer et al. guided the carbon flow from pyruvate to glycolysis back to G3P by overexpressing pps and deleting $p y k$ in E. coli [84]. In order to increase the carbon flux of isoprene in E. coli, Kim et al. knocked out nine genes related to byproduct formation (ackA-pta, poxB, ldhA, dld, adhE, pps, and atoDA) to obtain the strain ApsPtM, which produced $1832 \mathrm{mg} / \mathrm{L}$ of isoprene [85]. The various compounds that have a polyisoprene structure similar to MK-7 are listed 
as examples for future research on the construction of vitamin $\mathrm{K} 2$ producing microbial factories.

\section{Metabolic engineering of MK biosynthesis}

In B. subtilis, the biosynthesis of MK-7 proceeds via nine enzymatic reactions. The first six enzymes are encoded by the menFDHBEC operon and the menG gene is part of the hepS-menG-hepT operon, in which hepS/hepT encode all-trans heptaprenyl diphosphate synthase in the MEP pathway, while $m e n A$ and $m e n I$ are at separate loci in the genome [63]. Yang et al. [63] overexpressed each of the above cistrons using strong promoters in B. subtilis 168 , and the results revealed that only the step reaction by MenA, i.e. the prenylation of DHNA to DMK was a rate-limiting step in the MK-7 pathway. Cui et al. [64] boosted the synthesis of MK-7 precursors in B. subtilis by overexpressing menFBE, but the results indicated that the encoded enzymes do not catalyze a rate-limiting step of MK-7 biosynthesis, which was consistent with Yang's study mentioned before. Increasing the copy number of $m e n A$ at a different locus increased the titer of MK-7 by $42 \mathrm{mg} / \mathrm{L}$ compared with the original strain [64]. These results show that MenA is a critical enzyme that can determine the efficiency of the MK-7 synthesis pathway.

Although the futalosine-dependent pathway is widely investigated as a target for the development of new herbicides and antibiotics, there have been no studies on the metabolic engineering of the futalosine pathway to increase the yield of menaquinone [26, 29]. It may be a promising strategy for the biosynthesis of vitamin $\mathrm{K} 2$ to combine these two MK biosynthesis pathways.

\section{Vitamin $\mathrm{K} 2$ and membrane engineering}

The bilayer membrane is not only an interface between cell and the outside environment, but also contains intricate mechanism for signal transduction, transporters, cellular communication, adhesion, etc. [86, 87]. MK isoforms are lipid-soluble molecules that insert themselves into the bacterial cell membrane and shuttle electrons between the membrane-bound protein complexes in the electron-transport chain in many bacteria [41]. Hence, the synthesis of vitamin $\mathrm{K} 2$ may also affect by the state of the cell membrane, which has inspired studies of membrane engineering as a new direction for enhancing vitamin K2 biosynthesis.

Wang et al. [2] found that the stable surface tension and an optimized composition of the cell membrane are beneficial to the accumulation of MK-7. Furthermore, Cui et al. [88] showed that membrane components (GO:0006810, GO:0017000, GO:0008125, GO:0031224, GO:0051234) exhibited the most significantly changed expression levels in a comparative transcriptomic analysis of the intrinsic connections between biofilm formation and MK-7 production. Overexpression of cellmembrane-associated proteins such as signal receptors (BSU02010), transmembrane transporters (BSU29340, BSU03070), and tatAD-CD (BSU02630) could improve the synthesis of MK-7. These results directly indicate that the composition and homeostasis of the cell membrane can affect MK-7 synthesis. Co-expression of tatAD-CD (BSU02630) and menaquinol-cytochrome $\mathrm{c}$ reductase qcrA-C using the strong P43 promoter in the previously engineered strain BS20 increased the titer of MK-7 to $410 \mathrm{mg} / \mathrm{L}$ in shake-flask culture, which is the highest MK-7 titer reported to date $[64,88]$.

Similar to MK, lycopene, and carotenoids are biosynthesized from isoprene units and are accumulated in the cell membrane or neutral lipid droplets. Accordingly, there are reports that the yield of lycopene or carotenoids can be successfully improved by increasing the cellular lipid content (Fig. 3) [89, 90]. Coincidentally, $\mathrm{Hu}$ et al. [91] supplemented soybean oil to the medium, resulting in a maximal MK-7 yield of $40.96 \mathrm{mg} / \mathrm{L}$. Therefore, expanding the product spectrum will be useful to promote the metabolic engineering design-build-test-learn cycle and possibly increase the economic viability of microbial cell factories.

\section{Conclusions and prospects}

Traditionally, vitamin $\mathrm{K}$ is known for its essential role in the blood coagulation cascade and it is also involved in the maintenance of bone, preventing arterial hardening, modulating inflammation, and neuroprotection $[9,92]$. Among vitamin $\mathrm{K}$ isoforms, MK-7 has gained widespread attention because its dietary intake offers numerous health benefits due to its longer half-life in human blood compared to other isoforms $[15,22]$. With the progress of medical research, there is increasing evidence that the current recommended dietary supply of vitamin K2 cannot meet the needs of many individuals, and the market demand for vitamin K2 supplements is increasing significantly [9, 93-95].

Vitamin K2 plays a key role in the prokaryotic respiratory electron transport chain. Bacteria and archaea are the main producers of menaquinone [17, 96]. Different strains can produce different types of menaquinone. MK-7 is mainly produced by fermentation of B. subtilis, and its proportion among all isoforms can reach $90-96 \%$ [97]. Due to its long history of research and high vitamin $\mathrm{K} 2$ content, $B$. subtilis is considered the most promising potential strain for microbial production of MK-7. However, due to the long fermentation time and high industrial cost of $B$. subtilis, engineering $E$. coli to synthesize MK-7 is considered an attractive alternative for the economical industrial production of MK-7. 
Chemicals derived from isoprenoids and chorismate are highly valuable and can be applied in various industrial areas. Although many successful case studies offer hope for the industrial biosynthesis of MK-7, the obtainable titer are still too low. The inherent constraints of the MK-7 microbial factory are related to the complex and strictly regulated pathways including 37 enzymes, multiple cofactors, and a series of reactions from glucose. Firstly, the coordination of the different metabolic pathways leads to tradeoffs in menaquinone biosynthesis. For example, pyruvate and PEP are important precursors for the synthesis of the isoprene side chain and chorismate, respectively. But most PEP is converted into pyruvate, which needs to enter the TCA cycle to supply ATP and $\mathrm{NADH}$. Thus, balancing the fiuxes of PEP and pyruvate by designing dynamic regulatory is critical for the biosynthesis of different MK isoforms. The application of bifunctional pyruvate-responsive biosensor for dynamic dual control (activation and inhibition) to fine-tune the MK-7 yield is a promising approach for solving this problem [98]. In addition to coordinating complex pathways, it is also necessary to regulate the expression and activity of various enzymes, which also limits of vitamin K2 biosynthesis. Even if the same enzyme is expressed in chassis cells, the timing of expression and differences between chassis cells may have opposite effects on the yield of MK-7 (unpublished data). Another major bottleneck is the feedback inhibition of key enzymes, which also restricts the biosynthesis of vitamin K2. For example, DAHP synthase in the shikimate biosynthesis pathway is subject to feedback-inhibition by aromatic amino acids. The first committed enzyme of menaquinone biosynthesis in the human pathogen Mycobacterium tuberculosis (Mtb), MenD, is subject to feedback inhibition by direct binding of a downstream metabolite from the biosynthetic pathway, DHNA [99]. The allosteric binding sites of different bacterial MenD enzymes show limited conservation, and the regulation of the pathway by DHNA is limited to certain bacteria. Thus, feedback inhibition requires in-depth study for each newly discovered enzyme. Moreover, the rate-limiting enzymes, such as MenA, have low expression levels and the accumulation of HMBPP in the MEP pathway can significantly affected cell growth and isoprenoid production. Thus, balancing the metabolic flux of the biosynthetic enzymes is critical for vitamin K2 biosynthesis $[75,100]$.

The product spectrum related to vitamin $\mathrm{K} 2$ is a double-edged sword. Not only can it inspire metabolic engineering strategies of vitamin $\mathrm{K} 2$ biosynthesis, but it also means that related products in the cell compete for intermediate metabolites. For example, the (C5) isoprenoids DMAPP and IPP formed in either the MVA or the MEP pathway are condensed to form (C55) undecaprenyl diphosphate (UPP), which participates in cell wall biosynthesis. Therefore, the biosynthesis of UPP can compete with vitamin K2 synthesis for IPP and DMAPP, but blocking this process is bound to have a serious impact on cell growth [101]. Nevertheless, we can still learn from successful metabolic engineering strategies such as pathway engineering and membrane engineering from the product spectrum. In the past decade, the MEP pathway together with the MVA pathway and the shikimate route were engineered in microorganisms to produce exciting yields of downstream products. With the development of microbial biotechnology, MK-7 will become available at high production yields and low cost in the future.

Essentially, this review aims to provide an overview of the strategies for the microbial production of vitamin K2 based on the engineering of the shikimate pathway, polyisoprene biosynthesis and MK pathway. More importantly, we expand the spectrum of products similar to vitamin $\mathrm{K} 2$, which gives us more insights into available strain engineering strategies. Additionally, the intrinsic characteristics of the strain such as biofilm formation, cell membrane composition and electron transfer chain can provide inspiration for strategies that can enhance the production of MK-7. The utilization of more advanced biotechnological methods, such as dynamic regulation, computer-aided design of metabolic networks, and metabolic flux balance analysis will further increase the productivity and reduce the cost of microbial vitamin $\mathrm{K} 2$ fermentation to ultimately realize its industrial production.

\section{Abbreviations}

\section{Enzymes}

GlpK: Glycerol kinase; GlpD: Glycerol-3-phosphate dehydrogenase; TpiA:

Triosephosphate isomerase; Tkt: Transketolase; PykA: Pyruvate kinase II; AroA: 3-Deoxy-7-phosphoheptulonate synthase; AroB: 3-Dehydroquinate synthase; AroC: 3-Dehydroquinate dehydratase l; AroD: Shikimate dehydrogenase; AroK: Shikimate kinase; AroE: 3-Phosphoshikimate 1-carboxyvinyltransferase; AroF: Chorismate synthase; AtoB: Acetyl-CoA C-acetyltransferase; Erg10: AcetylCoA acetyl transferase; Erg13: Hydroxymethylglutaryl-CoA synthase; Hmg1/ Hmg2: Hydroxymethylglutaryl-CoA reductase; Erg12: Mevalonate kinase; Erg8: Phosphomevalonate kinase; Erg19: Mevalonate pyrophosphate decarboxylase; DxS: 1-Deoxyxylulose-5-phosphate synthase; DxR: 1-Deoxyxylulose5-phosphate reductoisomerase; IspD: 2-C-methylerythritol 4-phosphate cytidylyltransferase; IspE: 4-Diphosphocytidyl-2-C-methylerythritol kinase; IspF: 2-C-methylerythritol 2,4-cyclodiphosphate synthase; IspG: 4-Hydroxy3-methylbut-2-enyl diphosphate synthase; IspH: 4-Hydroxy-3-methylbut-2enyl diphosphate reductase; Idi: Isopentenyl-diphosphate delta-isomerase; IspA: Farnesyl diphosphate synthase; HepS/T: Heptaprenyl diphosphate synthase; MenF: Isochorismate synthase; MenD: 2-Succinyl-5-enolpyruvyl6-hydroxy-3-cyclohexene-1-carboxylate synthase; MenH: Demethylmenaquinone methyltransferase; MenC: O-Succinylbenzoate synthase; MenE: O-Succinylbenzoate-CoA ligase; MenB: 1,4-Dihydroxy-2-naphthoyl-CoA synthase; Menl: 1,4-Dihydroxy-2-naphthoyl-CoA hydrolase; MenA: 1,4-Dihydroxy-2-naphthoate heptaprenyltransferase; MenG: Demethylmenaquinone methyltransferase; MqnA: Chorismate dehydratase; MqnE: Aminodeoxyfutalosine synthase; MqnF: Aminodeoxyfutalosine deaminase; MqnB: Futalosine hydrolase; MqnC: Cyclic dehypoxanthinyl futalosine synthase; MqnD: 
1,4-Dihydroxy-6-naphthoate synthase; MTAN: Adenosylhomocysteine/aminodeoxyfutalosine nucleosidase; MvaE: Acetyl-CoA acetyltransferase/HMG-CoA reductase; MvaS: Hydroxymethylglutaryl-CoA synthase; MVK: Mevalonate kinase; UbiC: Chorismate lyase; UbiA: 4-Hydroxybenzoate octaprenyltransferase; HepPPS: Heptaprenyl pyrophosphate synthetase; mvaK1: Mevalonate kinase; mvaK2: Phosphomevalonate kinase; mvaD: Diphosphomevalonate decarboxylase; mvaA: Acetyl-CoA acetyltransferase; hsUBIAD1: Homo sapiens Geranylgeranyl diphosphate synthase.

\section{Metabolites}

Glu: Glucose; F6P: D-Fructose 6-phosphate; DAHP: Dihydroxyacetone phosphate; GAP: D-Glyceraldehyde 3-phosphate; E4P: Erythrose 4-phosphate; PEP: Phosphoenolpyruvate; PYR: Pyruvate; TCA cycle: Citrate cycle; CHA: Chorismate; DHAP: 3-Deoxy-darobino-heptulosonate 7-phosphate; DHQ: 3-Dehydroquinate; SA: Shikimate; DHS: 3-Dehydroshikimate; S3P: Shikimate 3-phosphate; EPSP: 5-Enolpyruvoylshikimate 3-phosphate; AcCoA: Acetyl-CoA; AACoA: Acetoacetyl-CoA; HMG-CoA: 3-Hydroxy-3-methyl-glutaryl-CoA; MVA: Mevalonate; MVAP: Mevalonate-5P; MVAPP: Mevalonate-5PP; DXP: 1-DeoxyD-xylulose 5-phosphate; MEP: 2-C-methyl-D-erythritol 4-phosphate; CDP-ME: 4-(Cytidine 5'-diphospho)-2-C-methyl-D-erythritol; CDP-MEP: 2-Phospho4-(cytidine 5'-diphospho)-2-C-methyl-D-erythritol; MECPP: 2-C-methyl-D-erythritol 2,4-cyclodiphosphate; HMBPP: 1-Hydroxy-2-methyl-2-butenyl 4-diphosphate; DMAPP: Dimethylallyl diphosphate; IPP: Isopentenyl diphosphate; GPP: Geranyl diphosphate; FPP: Farnesyl diphosphate; GGPP: Geranylgeranyl diphosphate; HPP: All-trans-Heptaprenyl diphosphate; ISoCHA: Isochorismate; SEPHCHC: 2-Succinyl-5-enolpyruvyl-6-hydroxy-3-cyclohexene-1-carboxylate; SHCHC: (1R,6R)-2-succinyl-6-hydroxy-2,4-cyclohexadiene-1-carboxylate; DHNA: 1,4-Dihydroxy-2-naphthoate; DMK-7: Demethylmenaqunol-7; OPP: All-trans-octaprenyl diphosphate; DPP: All-trans-decaprenyl diphosphate; MK: Menaquinone.

\section{Acknowledgements}

Not applicable.

\section{Authors' contributions}

$\mathrm{ZZ}$ and LL designed the review and wrote the manuscript. All authors read and approved the final manuscript.

\section{Funding}

This work was financially supported by the National Key R\&D Program of China (2019YFA0905300), the Tianjin Synthetic Biotechnology Innovation Capacity Improvement Project (TSBICIP-CXRC-004).

\section{Availability of data and materials}

Not applicable.

\section{Declarations}

\section{Ethics approval and consent to participate}

Not applicable.

\section{Consent for publication}

Not applicable.

\section{Competing interests}

The authors declare that they have no conflict of interest.

\section{Author details}

${ }^{1}$ School of Biological Engineering, Dalian Polytechnic University, Dalian 116034, China. ${ }^{2}$ Key Laboratory of Systems Microbial Biotechnology, Chinese Academy of Sciences, Tianjin 300308, China. ${ }^{3}$ Tianjin Institute of Industrial Biotechnology, Chinese Academy of Sciences, Tianjin 300308, China. ${ }^{4}$ National Technology Innovation Center of Synthetic Biology, Tianjin 300308, China. ${ }^{5}$ University of Chinese Academy of Sciences, Beijing 100049, China.

Received: 17 December 2020 Accepted: 2 April 2021

Published online: 13 April 2021

\section{References}

1. Song J, Liu H, Wang L, Dai J, Liu Y, Liu H, Zhao G, Wang P, Zheng Z. Enhanced production of vitamin K2 from Bacillus subtilis (natto) by mutation and optimization of the fermentation medium. Braz Arch Biol Technol. 2014;57:606-12.

2. Wang $H$, Liu $H$, Wang $L$, Zhao G, Tang $H$, Sun $X$, Ni W, Yang $Q$, Wang $\mathrm{P}$, Zheng Z. Improvement of menaquinone-7 production by Bacillus subtilis natto in a novel residue-free medium by increasing the redox potential. Appl Microbiol Biotechnol. 2019;103:7519-35.

3. Dam H. The antihemorrhagic vitamin of the chick:: occurrence and chemical nature. Nature. 1935;135:652-3.

4. Sato T, Inaba N, Yamashita T. MK-7 and its effects on bone quality and strength. Nutrients. 2020;12:965.

5. Capozzi A, Scambia G, Migliaccio S, Lello S. Role of vitamin K2 in bone metabolism: a point of view and a short reappraisal of the literature. Gynecol Endocrinol. 2020;36:285-8.

6. Halder M, Petsophonsakul P, Akbulut AC, Pavlic A, Bohan F, Anderson E, Maresz K, Kramann R, Schurgers L. Vitamin K: double bonds beyond coagulation insights into differences between vitamin $\mathrm{K} 1$ and $\mathrm{K} 2$ in health and disease. Int J Mol Sci. 2019;20:896.

7. Fusaro M, Cosmai L, Evenepoel P, Nickolas TL, Cheung AM, Aghi A, Tripepi G, Plebani M, lervasi G, Vettor R, Zaninotto M, Ravera M, Foramitti M, Giannini S, Sella S, Gallieni M. Vitamin K and kidney transplantation. Nutrients. 2020;12:2717.

8. Yu Y, Yu X, Cheng Q, Tang L, Shen M. The association of serum vitamin K2 levels with Parkinson's disease: from basic case-control study to big data mining analysis. Aging. 2020;12:16410-9.

9. Villa JKD, Diaz MAN, Pizziolo VR, Martino HSD. Effect of vitamin K in bone metabolism and vascular calcification: a review of mechanisms of action and evidences. Crit Rev Food Sci Nutr. 2017;57:3959-70.

10. Schwalfenberg GK. Vitamins K1 and K2: the emerging group of vitamins required for human health. J Nutr Metab. 2017;2017:6254836.

11. Berenjian A, Sarabadani Z. How menaquinone-7 deficiency influences mortality and morbidity among COVID-19 patients. Biocatal Agric Biotechnol. 2020;29:101792.

12. Wei H, Zhao G, Liu H, Wang H, Ni W, Wang P, Zheng Z. A simple and efficient method for the extraction and separation of menaquinone homologs from wet biomass of Flavobacterium. Bioprocess Biosyst Eng. 2018;41:107-13.

13. Tarento TDC, McClure DD, Talbot AM, Regtop HL, Biffin JR, Valtchev P, Dehghani F, Kavanagh JM. A potential biotechnological process for the sustainable production of vitamin K1. Crit Rev Biotechnol. 2018;39:1-19.

14. Vermeer C, van't Hoofd C, Knapen MHJ, Xanthoulea S. Synthesis of 2-methyl-1,4-naphthoquinones with higher gamma-glutamyl carboxylase activity than MK-4 both in vitro and in vivo. Bioorg Med Chem Lett. 2017;27:208-11.

15. Chatron N, Hammed A, Benoit E, Lattard V. Structural insights into phylloquinone (vitamin K1), menaquinone (MK4, MK7), and menadione (vitamin K3) binding to VKORC1. Nutrients. 2019;11:1-67.

16. Wu WJ, Gao H, Jin JS, Ahn BY. A comparatively study of menaquinone-7 isolated from Cheonggukjang with vitamin $\mathrm{K} 1$ and menaquinone-4 on osteoblastic cells differentiation and mineralization. Food Chem Toxicol. 2019:131:110540.

17. Ren L, Peng C, Hu X, Han Y, Huang H. Microbial production of vitamin K2: current status and future prospects. Biotechnol Adv. 2020;39:107453.

18. Novin D, van der Wel J, Seifan M, Berenjian A. The effect of aeration and mixing in developing a dairy-based functional food rich in menaquinone-7. Bioprocess Biosyst Eng. 2020;43:1773.

19. Xue J, Ahring BK. Enhancing isoprene production by genetic modification of the 1-deoxy-D-xylulose-5-phosphate pathway in Bacillus subtilis. Appl Environ Microbiol. 2011;77:2399-405.

20. Mahdinia E, Demirci A, Berenjian A. Strain and plastic composite support (PCS) selection for vitamin K (menaquinone-7) production in biofilm reactors. Bioprocess Biosyst Eng. 2017:40:1507-17.

21. Lal N, Berenjian A. Cis and trans isomers of the vitamin menaquinone-7: which one is biologically significant? Appl Microbiol Biotechnol. 2020;104:2765-76.

22. Marles RJ, Roe AL, Oketch-Rabah HA. US pharmacopeial convention safety evaluation of menaquinone-7, a form of vitamin K. Nutr Rev. 2017;75:553-78. 
23. Braasch-Turi M, Crans DC. Synthesis of naphthoquinone derivatives: menaquinones, lipoquinones and other vitamin K derivatives. Molecules. 2020;25:4477.

24. Bentley R, Meganathan R. Biosynthesis of vitamin K (menaquinone) in bacteria. Microbiol Rev. 1982;46:241-80.

25. Liu Y, Ding XM, Xue ZL, Hu LX, Zhang NJ, Wang Z, Yang JW, Cheng Q, Chen $\mathrm{MH}$, Zhang ZZ, Zheng ZM. The change of the state of cell membrane can enhance the synthesis of menaquinone in Escherichia coli. World J Microbiol Biotechnol. 2017:33:52.

26. Joshi S, Fedoseyenko D, Mahanta N, Manion H, Naseem S, Dairi T, Begley TP. Novel enzymology in futalosine-dependent menaquinone biosynthesis. Curr Opin Chem Biol. 2018;47:134-41.

27. Yokoyama K, Lilla EA. C-C bond forming radical SAM enzymes involved in the construction of carbon skeletons of cofactors and natural products. Nat Prod Rep. 2018;35:660-94.

28. Hein S, Klimmek O, Polly M, Kern M, Simon J. A class C radical S-adenosylmethionine methyltransferase synthesizes 8-methylmenaquinone. Mol Microbiol. 2017:104:449-62.

29. Zhi XY, Yao JC, Tang SK, Huang Y, Li HW, Li WJ. The futalosine pathway played an important role in menaquinone biosynthesis during early prokaryote evolution. Genome Biol Evol. 2014;6:149-60.

30. Mahanta N, Fedoseyenko D, Dairi T, Begley TP. Menaquinone biosynthesis: formation of aminofutalosine requires a unique radical SAM enzyme. J Am Chem Soc. 2013;135:15318-21.

31. Hiratsuka T, Furihata K, Ishikawa J, Yamashita H, Itoh N, Seto H, Dairi T. An alternative menaquinone biosynthetic pathway operating in microorganisms. Science. 2008;321:1670-3.

32. Seto H, Jinnai Y, Hiratsuka T, Fukawa M, Furihata K, Itoh N, Dairi T. Studies on a new biosynthetic pathway for menaquinone. J Am Chem Soc. 2008;130:5614-5.

33. Holland CK, Westfall CS, Schaffer JE, De Santiago A, Zubieta C, Alvarez S, Jez JM. Brassicaceae-specific Gretchen Hagen 3 acyl acid amido synthetases conjugate amino acids to chorismate, a precursor of aromatic amino acids and salicylic acid. J Biol Chem. 2019;294:16855-64.

34. Noda S, Shirai T, Oyama S, Kondo A. Metabolic design of a platform Escherichia coli strain producing various chorismate derivatives. Metab Eng. 2016;33:119-29.

35. Mahanta N, Hicks KA, Naseem S, Zhang Y, Fedoseyenko D, Ealick SE, Begley TP. Menaquinone biosynthesis: biochemical and structural studies of chorismate dehydratase. Biochemistry. 2019;58:1837-40.

36. Dairi T. Menaquinone biosyntheses in microorganisms. Methods Enzymol. 2012;515:107-22

37. LiY, Wang G. Strategies of isoprenoids production in engineered bacteria. J Appl Microbiol. 2016;121:932-40.

38. Liao P, Hemmerlin A, Bach TJ, Chye ML. The potential of the mevalonate pathway for enhanced isoprenoid production. Biotechnol Adv. 2016:34:697-713.

39. Rohmer M. The discovery of a mevalonate-independent pathway for isoprenoid biosynthesis in bacteria, algae and higher plants. Nat Prod Rep. 1999;16:565-74.

40. Johnston JM, Bulloch EM. Advances in menaquinone biosynthesis: sublocalisation and allosteric regulation. Curr Opin Struct Biol. 2020;65:33-41.

41. Kawamukai M. Biosynthesis and applications of prenylquinones. Biosci Biotechnol Biochem. 2018:82:963-77.

42. Boe CA, Holo H. Engineering Lactococcus lactis for increased vitamin $\mathrm{K} 2$ production. Front Bioeng Biotechnol. 2020;8:191.

43. Hirota Y, Suhara Y. New aspects of Vitamin $K$ research with synthetic ligands: transcriptional activity via SXR and neural differentiation activity. Int J Mol Sci. 2019;20:3006.

44. Yuan P, Cui S, Liu Y, Li J, Lv X, Liu L, Du G. Combinatorial engineering for improved menaquinone-4 biosynthesis in Bacillus subtilis. Enzyme Microb Technol. 2020;141:109652.

45. Sun X, Liu H, Wang P, Wang L, Ni W, Yang Q, Wang H, Tang H, Zhao G, Zheng Z. Construction of a novel MK-4 biosynthetic pathway in Pichia pastoris through heterologous expression of HsUBIAD1. Microb Cell Fact. 2019;18:169.

46. Kong MK, Lee PC. Metabolic engineering of menaquinone-8 pathway of Escherichia coli as a microbial platform for vitamin $\mathrm{K}$ production. Biotechnol Bioeng. 2011;108:1997-2002.
47. Simes DC, Viegas CSB, Araújo N, Marreiros C. Vitamin K as a diet supplement with impact in human health: current evidence in age-related diseases. Nutrients. 2020;12:138.

48. Shearer MJ, Newman P. Recent trends in the metabolism and cell biology of vitamin $\mathrm{K}$ with special reference to vitamin K cycling and MK-4 biosynthesis. J Lipid Res. 2014;55:345-62.

49. Schurgers L, Teunissen K, Marjo KH. Vitamin K-containing dietary supplements: comparison of synthetic vitamin $\mathrm{K} 1$ and natto-derived menaquinone-7. Blood. 2007;109:3279-83.

50. Berenjian A, Mahanama R, Talbot A, Regtop H, Kavanagh J, Dehghani F. Advances in menaquinone-7 production by Bacillus subtilis natto: fedbatch glycerol addition. Am J Biochem Biotechnol. 2012;8:105-10.

51. Sumi H. Accumulation of vitamin K (menaquinone7) in plasma after ingestion of natto and natto Bacilli (B. subtilis natto). Food Sci Technol Res. 1999;5:48-50.

52. Sato T, Yamada Y, Ohtani Y, Mitsui N, Murasawa H, Araki S. Production of menaquinone (vitamin K2)-7 by Bacillus subtilis. J Biosci Bioeng. 2001;91:16-20.

53. Tsukamoto $Y$, Ichise $H$, Kakuda H, Yamaguchi M. Intake of fermented soybean (natto) increases circulating vitamin K2 (menaquinone-7) and gamma-carboxylated osteocalcin concentration in normal individuals. J Bone Miner Metab. 2000;18:216-22.

54. Luo M-M, Ren L-J, Chen S-L, Ji X-J, Huang H. Effect of media components and morphology of Bacillus natto on menaquinone-7 synthesis in submerged fermentation. Biotechnol Bioproc Eng. 2017;21:777-86.

55. Mahdinia E, Demirci A, Berenjian A. Optimization of Bacillus subtilis natto growth parameters in glycerol-based medium for vitamin $\mathrm{K}$ (menaquinone-7) production in biofilm reactors. Bioprocess Biosyst Eng. 2018;41:195-204

56. Lee JH, Wendisch VF. Biotechnological production of aromatic compounds of the extended shikimate pathway from renewable biomass. J Biotechnol. 2017;257:211-21.

57. Tzin V, Galili G. New insights into the shikimate and aromatic amino acids biosynthesis pathways in plants. Mol Plant. 2010;3:956-72.

58. Jiang $\mathrm{M}$, Zhang $\mathrm{H}$. Engineering the shikimate pathway for biosynthesis of molecules with pharmaceutical activities in E. coli. Curr Opin Biotechnol. 2016:42:1-6.

59. Ikeda M. Towards bacterial strains overproducing L-tryptophan and other aromatics by metabolic engineering. Appl Microbiol Biotechnol. 2006:69:615-26.

60. Kim B, Binkley R, Kim HU, Lee SY. Metabolic engineering of Escherichia coli for the enhanced production of L-tyrosine. Biotechnol Bioeng. 2018;115:2554-64.

61. Chen L, Zeng AP. Rational design and metabolic analysis of Escherichia coli for effective production of L-tryptophan at high concentration. Appl Microbiol Biotechnol. 2017;101:559-68.

62. Liu X, Niu H, Li Q, Gu P. Metabolic engineering for the production of L-phenylalanine in Escherichia coli. 3 Biotech. 2019;9:85.

63. Yang S, Cao Y, Sun L, Li C, Lin X, Cai Z, Zhang G, Song H. Modular pathway engineering of Bacillus subtilis to promote de novo biosynthesis of menaquinone-7. ACS Synth Biol. 2019;8:70-81.

64. Cui S, Lv X, Wu Y, Li J, Du G, Ledesma-Amaro R, Liu L. Engineering a bifunctional Phr60-Rap60-Spo0A quorum-sensing molecular switch for dynamic fine-tuning of menaquinone-7 synthesis in Bacillus subtilis. ACS Synth Biol. 2019;8:1826-37.

65. Kim H, Kim SY, Sim GY, Ahn JH. Synthesis of 4-hydroxybenzoic acid derivatives in Escherichia coli. J Agric Food Chem. 2020;68:9743-9.

66. Choi S, Lee HN, Park E, Lee SJ, Kim ES. Recent advances in microbial production of cis, cis-muconic acid. Biomolecules. 2020;10:1238.

67. Rekhter D, Lüdke D, Ding Y, Feussner K, Zienkiewicz K, Lipka V, Wiermer $M, Z$ hang $Y$, Feussner I. Isochorismate-derived biosynthesis of the plant stress hormone salicylic acid. Science. 2019;365:498-502.

68. Noda S, Kondo A. Recent advances in microbial production of aromatic chemicals and derivatives. Trends Biotechnol. 2017;35:785-96.

69. Lin Y, Shen X, Yuan Q, Yan Y. Microbial biosynthesis of the anticoagulant precursor 4-hydroxycoumarin. Nat Commun. 2013;4:2603.

70. Wang C, Zada B, Wei G, Kim SW. Metabolic engineering and synthetic biology approaches driving isoprenoid production in Escherichia coli. Bioresour Technol. 2017;241:430-8.

71. Frank A, Groll M. The methylerythritol phosphate pathway to isoprenoids. Chem Rev. 2017;117:5675-703. 
72. Kuzuyama T. Biosynthetic studies on terpenoids produced by Streptomyces. J Antibiot (Tokyo). 2017;70:811-8.

73. Zada B, Wang C, Park JB, Jeong SH, Park JE, Singh HB, Kim SW. Metabolic engineering of Escherichia coli for production of mixed isoprenoid alcohols and their derivatives. Biotechnol Biofuels. 2018;11:210.

74. Tetali SD. Terpenes and isoprenoids: a wealth of compounds for global use. Planta. 2019;249:1-8.

75. Ma Y, McClure D, Somerville M, Proschogo N, Dehghani F, Kavanagh $J$, Coleman N. Metabolic engineering of the MEP pathway in Bacillus subtilis for increased biosynthesis of menaquinone-7. ACS Synth Biol. 2019;8:1620-30.

76. Chen T, Xia H, Cui S, Lv X, Li X, Liu Y, Li J, Du G, Liu L. Combinatorial methylerythritol phosphate pathway engineering and process optimization for increased menaquinone-7 synthesis in Bacillus subtilis. Microbiol Biotechnol. 2020;30:762-9.

77. Gao Q, Chen H, Wang W, Huang J, Tao Y, Lin B. Menaquinone-7 production in engineered Escherichia coli. World J Microbiol Biotechnol. 2020;36:132.

78. Wraight CA. The role of quinones in bacterial photosynthesis. Photochem Photobiol. 2010;30:767-76.

79. Rubat S, Varas I, Sepulveda R, Almonacid D, Gonzalez-Nilo F, Agosin E. Increasing the intracellular isoprenoid pool in Saccharomyces cerevisiae by structural fine-tuning of a bifunctional farnesyl diphosphate synthase. FEMS Yeast Res. 2017;17:fox032. https://doi.org/10.1093/femsyr/ fox032.

80. Meadows CW, Kang A, Lee TS. Metabolic engineering for advanced biofuels production and recent advances toward commercialization. Biotechnol J. 2018;13:1600433

81. Collins M, Jones D. Distribution of isoprenoid quinone structural types in bacteria and their taxonomic implications. Microbiol Rev. 1981:45:316-54

82. Kwak S, Kim SR, Xu H, Zhang GC, Lane S, Kim H, Jin YS. Enhanced isoprenoid production from xylose by engineered Saccharomyces cerevisiae. Biotechnol Bioeng. 2017;114:2581-91.

83. George KW, Thompson MG, Kim J, Baidoo EEK, Wang G, Benites VT, Petzold CJ, Chan LJG, Yilmaz S, Turhanen P, Adams PD, Keasling JD, Lee TS. Integrated analysis of isopentenyl pyrophosphate (IPP) toxicity in isoprenoid-producing Escherichia coli. Metab Eng. 2018:47:60-72.

84. Farmer W, Liao JC. Precursor balancing for metabolic engineering of lycopene production in Escherichia coli. Biotechnol Progr. 2001;17:57-61.

85. Kim JH, Wang C, Jang HJ, Cha MS, Park JE, Jo SY, Choi ES, Kim SW. Isoprene production by Escherichia coli through the exogenous mevalonate pathway with reduced formation of fermentation byproducts. Microb Cell Fact. 2016;15:214.

86. Saeui C, Mathew M, Liu L, Urias E, Yarema K. Cell surface and membrane engineering: emerging technologies and applications. J Funct Biomater. 2015;6:454-85.

87. Kang MK, Tullman-Ercek D. Engineering expression and function of membrane proteins. Methods. 2018;147:66-72.
88. Cui S, Xia H, Chen T, GuY, Lv X, LiuY, Li J, Du G, Liu L Cell membrane and electron transfer engineering for improved synthesis of menaquinone-7 in Bacillus subtilis. iscience. 2020;23:100918.

89. Hong J, Park SH, Kim S, Kim SW, Hahn JS. Efficient production of lycopene in Saccharomyces cerevisiae by enzyme engineering and increasing membrane flexibility and NAPDH production. Appl Microbiol Biotechnol. 2019:103:211-23.

90. Wang C, Zhao S, Shao X, Park JB, Jeong SH, Park HJ, Kwak WJ, Wei G, Kim SW. Challenges and tackles in metabolic engineering for microbial production of carotenoids. Microb Cell Fact. 2019;18:55.

91. Hu XC, Liu WM, Luo MM, Ren LJ, Ji XJ, Huang H. Enhancing menaquinone-7 production by Bacillus natto R127 through the nutritional factors and surfactant. Appl Biochem Biotechnol. 2017;182:1630-41.

92. Myneni VD, Mezey E. Regulation of bone remodeling by vitamin K2 Oral Dis. 2017;23:1021-8.

93. Shea MK, Booth SL. Update on the role of vitamin K in skeletal health. Nutr Rev. 2008;66:549-57.

94. Schurgers L, Cranenburg E, Vermeer C. Vitamin K: the coagulation vitamin that became omnipotent. Thromb Haemost. 2007;98:120-5.

95. Vermeer $\mathrm{C}$. Vitamin $\mathrm{K}$ : the effect on health beyond coagulation - an overview. Food Nutr Res. 2012;56:5329.

96. Walther B, Karl JP, Booth SL, Boyaval P. Menaquinones, bacteria, and the food supply: the relevance of dairy and fermented food products to vitamin K requirements. Adv Nutr. 2013;4:463-73.

97. Sato T, Yamada Y, Ohtani Y, Mitsui N, Murasawa H, Araki S. Production of menaquinone (vitamin K2)-7 by Bacillus subtilis. J Biosci Bioeng. 2001;91:16-20.

98. Xu X, Li X, Liu Y, Zhu Y, Li J, Du G, Chen J, Ledesma-Amaro R, Liu L. Pyruvate-responsive genetic circuits for dynamic control of central metabolism. Nat Chem Biol. 2020;16:1261-8.

99. Bashiri G, Nigon LV, Jirgis ENM, Ho NAT, Stanborough T, Dawes SS, Baker EN, Bulloch EMM, Johnston JM. Allosteric regulation of menaquinone (vitamin K2) biosynthesis in the human pathogen Mycobacterium tuberculosis. J Biol Chem. 2020;295:3759-70.

100. Li Q, Fan F, Gao X, Yang C, Bi C, Tang J, Liu T, Zhang X. Balanced activation of IspG and IspH to eliminate MEP intermediate accumulation and improve isoprenoids production in Escherichia coli. Metab Eng. 2017:44:13-21.

101. Wang Y, Desai J, Zhang Y, Malwal SR, Shin CJ, Feng X, Sun H, Liu G, Guo RT, Oldfield E. Bacterial cell growth inhibitors targeting undecaprenyl diphosphate synthase and undecaprenyl diphosphate phosphatase. ChemMedChem. 2016;11:2311-9.

\section{Publisher's Note}

Springer Nature remains neutral with regard to jurisdictional claims in published maps and institutional affiliations.

Ready to submit your research? Choose BMC and benefit from

- fast, convenient online submission

- thorough peer review by experienced researchers in your field

- rapid publication on acceptance

- support for research data, including large and complex data types

- gold Open Access which fosters wider collaboration and increased citations

- maximum visibility for your research: over $100 \mathrm{M}$ website views per year

At $\mathrm{BMC}$, research is always in progress.

Learn more biomedcentral.com/submissions 